\title{
Rescue effect of lipid emulsion on bupivacaine-induced cardiac toxicity in cardiomyocytes
}

\author{
LIBIN YANG ${ }^{1,2}$, ZHIXIA BAI ${ }^{2}$, DANNI LV ${ }^{1}$, HAIBO LIU $^{1}$, XIAOHUI LI $^{1}$ and XUEXIN CHEN ${ }^{2}$ \\ ${ }^{1}$ Clinical Medicine School, Ningxia Medical University; ${ }^{2}$ Department of Anesthesiology, \\ Tumor Hospital of the General Hospital of Ningxia Medical University, Yinchuan, Ningxia 750004, P.R. China
}

Received November 5, 2014; Accepted May 14, 2015

DOI: $10.3892 / \mathrm{mmr} .2015 .3852$

\begin{abstract}
The aim of the present study was to investigate the mechanism underlying the rescue effect of lipid emulsion on bupivacaine (BPV)-induced cardiomyocyte toxicity. The inhibitory effects of BPV on $\mathrm{H} 9 \mathrm{c} 2$ myoblast cell proliferation were investigated using an MTT assay. The H9c2 myoblast cells were treated with either $1 \mathrm{mM}$ BPV or $1 \%$ lipid emulsion (LE) alone, or co-treated with both of the drugs. Cell apoptosis was detected using both Annexin V/propidium iodide staining and a terminal deoxynucleotidyl transferase dUTP assay. The protein expression levels of apoptosis-associated proteins were quantified using western blot analysis, and the mRNA expression levels were quantified by reverse transcription-quantitative polymerase chain reaction. The expression levels of reactive oxidative species, malondialdehyde, superoxide dismutase, and catalase were quantified using the optical density values obtained from a spectrophotometer. In addition, the mechanism underlying the mitochondrial function of the H9c2 myoblast cells was investigated using both JC-1 staining, and cyclosporin A and atractyloside treatment. The results indicated that the $\mathrm{H} 9 \mathrm{c} 2$ myoblast cells treated with BPV exhibited significantly higher levels of apoptosis. Furthermore, BPV treatment increased the levels of oxidative stress, and caused mitochondrial dysfunction within the $\mathrm{H} 9 \mathrm{c} 2$ myoblast cells. LE treatment reversed the effects of BPV treatment in the $\mathrm{H} 9 \mathrm{c} 2$ myoblast cells.
\end{abstract}

\section{Introduction}

Bupivacaine (BPV) is a sodium channel blocker commonly used as a local anesthetic in order to reduce postoperative pain. In addition, patients undergoing laparoscopic surgery

Correspondence to: Professor Xuexin Chen, Department of Anesthesiology, Tumor Hospital of the General Hospital of Ningxia Medical University, 804 Shengli South Street, Yinchuan, Ningxia 750004, P.R. China

E-mail: chenxuexin666@sohu.com

Key words: bupivacaine, lipid emulsion, cardiac toxicity, H9c2 cells, cyclosporin, atractyloside may be administered high doses of BPV as a postoperative analgesic (1). Despite its widespread usage in clinical settings, BPV may also cause rapid cardiovascular collapse. Two mechanisms underlying BPV-induced cardiotoxicity have been proposed: (i) Cardiotoxicity caused by blockage of the sodium, calcium, and potassium ion channels, or (ii) cardiotoxicity caused by BPV interference with mitochondrial energy metabolism (2-4).

The mitochondrial apoptosis pathway is one of the primary cellular processes that lead to cell death. An important factor in mitochondrial apoptosis is the mitochondrial permeability transition pore (mPTP). mPTP is a non-specific pore located in the inner mitochondrial membrane, which opens in the presence of elevated calcium concentrations, specifically when these elevated calcium concentrations are accompanied by high levels of oxidative stress, and depleted levels of adenine nucleotides (5). Opening of the MPTP causes alterations in the ionic balance, which in turn leads to destruction of the inner mitochondrial membrane, and release of cytochrome $c$ and apoptosis-inducing factors into the cytosol. These factors result in cell death via caspase-dependent or independent cascade reactions $(6,7)$.

Elevated levels of mitochondrial calcium are responsible for the opening of the MPTP (8). However, oxidative stress may induce mPTP opening by increasing the sensitivity of the pore to calcium (8). Destabilization of the ion concentration within the mitochondrial membrane in turn leads to apoptotic cell death. When cells are under oxidative stress, the balance between the production and the elimination of reactive oxygen species (ROS) is deregulated. The levels of ROS within the cell have a significant role in cell survival. High levels of ROS induce DNA damage and abnormal protein expression, which ultimately lead to cell death (9). Another biological marker of oxidative stress is malondialdehyde (MDA), which is a product of polyunsaturated fatty acid peroxidation. MDA has a long half-life and is highly reactive, which allows it to interact with biomolecules such as nucleic acids and proteins, often causing irreversibly damage to cellular function (10). The antioxidant defense mechanisms that eliminate ROS are crucial for protecting the organism against oxidative damage. Numerous enzymes exhibit antioxidative properties, including superoxide dismutase (SOD) and catalase (CAT) (11). SOD carries out its protective action by catalyzing the transformation of reactive oxygen radicals into hydrogen peroxide and oxygen. 
CAT in turn reduces hydrogen peroxide into non-toxic water and oxygen (12). The balance between SOD and CAT activity is crucial to the maintenance of a steady-state between toxic superoxide radicals and hydrogen peroxide (13).

Until recently, a cardiopulmonary bypass was the only method shown to be effective in the treatment of refractory cardiac arrest caused by local anesthetic overdose (14). Previous studies have demonstrated that treatment with lipid emulsion (LE) attenuates BPV-induced toxicity in various animal models $(15,16)$, and in the rat heart (17). Two hypotheses have been proposed to explain the reversal effects of LE on BPV-induced toxicity: (i) Lipid sink theory, or (ii) metabolic theory. The lipid sink theory hypothesizes that the lipophilic molecules contained within the local anesthetic partition into a lipemic plasma compartment making them inaccessible to the surrounding tissue, which in turn leads to an increase in the concentration of local anesthetic within the plasma (18). However, Litz et al (19) demonstrated that lipid infusion rapidly reduced serum anesthetic levels, which raises doubt regarding the "lipid sink" hypothesis. The metabolic theory hypothesizes that BPV inhibits fatty acid transport within the inner mitochondrial membrane, and that the lipids prevent the metabolites from being oxidized (20).

Although there have been numerous successful clinical reports of LE attenuation of BPV-induced cardiac arrest, the molecular mechanism underlying the rescue effects of LE has yet to be elucidated. The present study used $\mathrm{H} 9 \mathrm{c} 2$ rat neonatal myoblast cells to investigate BPV-induced cardiotoxicity, and to elucidate the mechanisms underlying the rescue effects of LE.

\section{Materials and methods}

Drugs and reagents. The 20\% LE (C14-24; production batch 13010841) was purchased from Libang Pharmaceutical Co., Ltd. (Xi'an, China), and adjusted to a final concentration of $1 \%$ using Dulbecco's modified Eagle's medium (DMEM). The BPV hydrochloride (production batch 121120) was purchased from Wuhu Kangqi Pharmaceutical Co., Ltd. (Wuhu, China), and adjusted to a final concentration of $1 \mathrm{mM}$ using complete DMEM medium. Cyclosporin A (CsA) was purchased from Sigma-Aldrich (St. Louis, MO, USA), and was dissolved in dimethyl sulfoxide (DMSO) prior to cellular treatment at a final concentration of $1 \mu \mathrm{M}$. Atractyloside (Atr) was purchased from Nanjing Spring \& Autumn Biological Engineering Co., Ltd. (Nanjing, China). Atr purity (>98\%) was determined using high performance liquid chromatography, and dissolved in DMSO prior to cellular treatment at a final concentration of $1 \mu \mathrm{M}$. Fetal bovine serum (FBS), DMEM, penicillin, and streptomycin were purchased from Gibco Life Technologies (Carlsbad, CA, USA). DMSO and the MTT assay were purchased from Sigma-Aldrich. All primary and secondary antibodies were obtained from Cell Signaling Technology, Inc. (Danvers, MA, USA). The MDA, CAT, and dichloro-dihydro-flurorescein diacetate (DCFH-DA) ROS detection kits were obtained from Beyotime Institute of Biotechnology (Jiangsu, China). The terminal deoxynucleotidyl transferase-mediated biotinylated dUTP nick end labeling (TUNEL) kit was purchased from Roche Diagnostics Co. (Indianapolis, IN, USA).
Cell culture. The H9c2 rat neonatal myoblast cells were obtained from the American Type Culture Collection (Manassas, VA, USA). The cells were maintained in high glucose DMEM supplemented with $10 \%(\mathrm{v} / \mathrm{v})$ heat-inactivated FBS, $100 \mathrm{U} / \mathrm{ml}$ penicillin, and $100 \mu \mathrm{g} / \mathrm{ml}$ streptomycin at $37^{\circ} \mathrm{C}$ in an atmosphere containing $5 \% \mathrm{CO}_{2}$. Once the cells had reached $70-80 \%$ confluence, the media was replaced with DMEM containing 1\% LE (LE group), DMEM containing $1 \mathrm{mM}$ BPV (BPV group), DMEM containing $1 \mathrm{mM}$ BPV and $1 \% \mathrm{LE}(\mathrm{BPV}+\mathrm{LE})$, or with fresh DMEM (control group).

Cell viability assay. Cell viability was determined using an MTT assay. Briefly, the H9c2 myoblast cells were seeded into 96-well plates at a density of $5 \times 10^{3} /$ well. A total of $1 \mathrm{mM} \mathrm{BPV}$ was added to the wells, and the cells were subsequently incubated for $0,12,24$, and $36 \mathrm{~h}$. A total of $10 \mu \mathrm{l}$ MTT solution in $5 \mathrm{mg} / \mathrm{ml}$ phosphate-buffered saline (PBS) was then added into each well, and the cells were incubated at $37^{\circ} \mathrm{C}$ for a further $4 \mathrm{~h}$. The medium was discarded, following which $100 \mu \mathrm{l}$ DMSO was added to each well, and the plates were gently agitated for $5 \mathrm{~min}$. Optical density was determined at $590 \mathrm{~nm}$ using a Thermo MK3 microplate spectrophotometer (Thermo Fisher Scientific, Inc., Waltham, MA, USA) (21). Cellular absorbance in the absence of treatment was determined as $100 \%$, indicating cell survival. Each treatment was repeated four times, and each experiment was performed in triplicate.

Intracellular ROS assay. ROS levels were measured using the non-fluorescent DCFH-DA probe as previously described (22). DCFH-DA passively diffuses into cells where it is deacetylated by esterases in order to form non-fluorescent 2'-7'-dichlorofluorescein (DCFH). In the presence of ROS, DCFH reacts to form the fluorescent product dichlorofluorescein, which remains within the cells. Briefly, the H9c2 myoblast cells were seeded at a density of $1 \times 10^{5} /$ well into 96 -well plates. Following a period of $24 \mathrm{~h}$, the culture wells were treated with various treatment solutions for $24 \mathrm{~h}$, and the cells were washed three times with phosphate-buffered saline. DCFH-DA, diluted to a final concentration of $10 \mu \mathrm{M}$ in DMEM without FBS, was added to the cell cultures, which were then incubated for $20 \mathrm{~min}$ at $37^{\circ} \mathrm{C}$. Cellular fluorescence was measured at $485 \mathrm{~nm}$ excitation and $530 \mathrm{~nm}$ emission using a Safire 2 microplate reader (Tecan Schweiz, Uetikon, Switzerland). An increase in the fluorescence values was determined as an increase in intracellular ROS, as compared with the control.

Total SOD (T-SOD) enzymatic activity assay. T-SOD activity was determined using a WST T-SOD Assay kit (Dojindo Molecular Technologies, Inc., Kumamoto, Japan), according to the manufacturer's instructions (23). The T-SOD assay uses highly water-soluble WST-1 tetrazolium salts that react with superoxide anions $\left(\mathrm{O}^{2-}\right)$ in order to produce a water-soluble formazan dye. The H9c2 cells were seeded at a density of $1 \times 10^{5} /$ well into 96 -well plates. Following a period of $24 \mathrm{~h}$ the cells were treated with $1 \mathrm{mM} \mathrm{BPV}, 1 \% \mathrm{LE}$, or a combination of $1 \mathrm{mM}$ BPV and $1 \% \mathrm{LE}$ for $24 \mathrm{~h}$. The cells were then washed three times with PBS, and the samples were tested, and compared against a standard curve ranging from 2-200 U/ml. The colorimetric assay is performed by measuring the levels of formazan produced by the reaction between WST-1 and $\mathrm{O}^{2-}$. 
The reaction reduction rate is linearly correlated to xanthine oxidase activity, which is inhibited by T-SOD. Cellular absorbance was measured using a microplate reader (Safire2; Tecan Schweiz AG, Männedorf, Switzerland) set to $450 \mathrm{~nm}$.

Lipid peroxidation assay. A lipid peroxidation assay was carried out using a commercial kit according to the manufacturer's instructions, in order to quantify cellular MDA. Briefly, the H9c2 myoblast cells were harvested by trypsinization (Gibco Life Technologies), and the cellular extracts were subsequently prepared by sonication at $950 \mathrm{~W}, 30 \%$ for $10 \mathrm{~min}, 1 \mathrm{sec}$ with $2 \mathrm{sec}$ interval, in ice-cold buffer containing $50 \mathrm{mM}$ Tris- $\mathrm{HCl} \mathrm{pH} 7.5,5 \mathrm{mM}$ EDTA, and $1 \mathrm{mM}$ dithiothreitol. Following sonication, the cells were centrifuged at $10,000 \mathrm{x} \mathrm{g}$ for $20 \mathrm{~min}$ in order to remove any debris. The supernatant was then used to measure MDA levels and protein content (24). MDA content was quantified by thiobarbituric acid assay, with 1,1,3,3-tetramethoxypropane as an external standard. The optical density was measured at $532 \mathrm{~nm}$ using a microplate reader (Safire2; Tecan Schweiz AG), according to the manufacturer's instructions, and the protein content was determined using the Bradford method (Nanjing Jiancheng Bioengineering Institute, Nanjing, China).

CAT specific activity. The $\mathrm{H} 9 \mathrm{c} 2$ myoblast cell CAT activity was detected using a CAT analysis kit (Beyotime Institute of Biotechnology), according to the manufacturer's instructions (25). Briefly, the cells were treated with hydrogen peroxide and incubated at $25^{\circ} \mathrm{C}$ for $1-5 \mathrm{~min}$ so that the hydrogen peroxide could react with CAT. Following treatment, the remaining hydrogen peroxide was coupled with a substrate prior to further treatment with peroxidase, in order to produce N-4-antipyryl-3-chloro-5-sulfonate-benzoquinon emonoimine, which has a maximum absorption of $520 \mathrm{~nm}$. Cellular absorption was quantified spectrophotometrically (Safire2; Tecan Schweiz AG). CAT activity was subsequently calculated from the assay results, and protein concentration was measured using a Bradford Protein Assay kit (Beyotime Institute of Biotechnology).

H9c2 Annexin V-fluorescein isothiocyanate (FITC)/propidium iodide (PI) apoptosis assay. The apoptotic rate of the $\mathrm{H} 9 \mathrm{c} 2$ cells was detected by flow cytometry using the Annexin V-FITC/PI double-labeling method. The cells $\left(1 \times 10^{5}\right.$ cells $\left./ \mathrm{ml}\right)$ were seeded in 6-well plates, and were treated with $1 \mathrm{mM} \mathrm{BPV}$ and $1 \% \mathrm{LE}$, as described above. The cells were then trypsinized and collected by centrifugation at $160 \mathrm{x}$ g for $5 \mathrm{~min}$. After washing with PBS, the cells were double-stained with an Annexin V-FITC Apoptosis Detection kit (eBioscience, San Diego, CA, USA), according to the manufacturer's instructions. Briefly, the cells were resuspended in Annexin V-FITC binding buffer, and incubated with Annexin V-FITC $(1 \mu \mathrm{g} / \mathrm{ml})$ for $15 \mathrm{~min}$ at room temperature in the dark, followed by an incubation with PI $(10 \mu \mathrm{g} / \mathrm{ml})$ for $15 \mathrm{~min}$. Samples were analyzed using a flow cytometer (FACSCalibur; BD Biosciences, Mountain View, CA, USA) by two parameter dot-plots. A total of 10,000 cells were recorded in each case.

H9c2 myoblast cell apoptosis assay. H9c2 cell apoptosis was detected using a TUNEL kit (26). Briefly, the H9c2 myoblast cells were cultured on cover slips at a density of $1 \times 10^{5} / \mathrm{ml}$ for $24 \mathrm{~h}$. Following treatment, the cells were fixed in $4 \%$ paraformaldehyde for $30 \mathrm{~min}$ at room temperature. The cells were then incubated with a methanol solution supplemented with $0.3 \%$ hydrogen peroxide for $30 \mathrm{~min}$ at room temperature, prior to further incubation with a permeabilizing solution supplemented with $0.1 \%$ sodium citrate and $0.1 \%$ Triton X-100 (Biosharp, Nanjing, China) for $2 \mathrm{~min}$ at $4^{\circ} \mathrm{C}$. The cells were subsequently incubated with the TUNEL reaction mixture for $60 \mathrm{~min}$ at $37^{\circ} \mathrm{C}$, and visualized by inverted fluorescence microscopy (AF6000; Leica Microsystems GmbH, Wetzlar, Germany). The TUNEL-positive nuclei were counted in four non-overlapping fields per cover slip, and the total percentage of TUNEL-positive nuclei was then calculated by determining the ratio of TUNEL-positive counts to the total number of nuclei, as determined by DAPI 33342 (Beyotime Institute of Biotechnology) counterstaining.

Western blot analysis. Proteins were extracted from the H9c2 myoblast cells using a radioimmunoprecipitation buffer supplemented with $1 \mathrm{mM}$ phenylmethylsulfonyl fluoride (Beyotime Institute of Biotechnology). The protein concentrations were determined using the Bradford Protein Assay kit. The protein samples $(50 \mu \mathrm{g})$ were resuspended in sample buffer containing $2 \%$ SDS, $2 \% \beta$-mercaptoethanol, 50 mmol/1 Tris- $\mathrm{HCl} \mathrm{pH} 6.8$, $10 \%$ glycerol, and $0.05 \%$ bromophenol blue. The proteins were separated by $15 \%$ SDS-PAGE and transferred to polyvinylidene fluoride membranes (Merck Millipore, Bedford, UK). Following membrane blockage with Tris-buffered saline containing $0.1 \%$ Tween ${ }^{\circledR} 20$ (TBST) supplemented with $2.5 \%$ bovine serum albumin (Beijing Solarbio Science \& Technology, Co., Ltd., Beijing, China) for $1 \mathrm{~h}$ at room temperature, the membranes were washed twice with TBST prior to being incubated with the following primary antibodies: Anti-B-cell lymphoma 2 (Bcl-2; 50E3, cat. no. 2870, 1:1,000), anti-Bcl-2-associated X protein (Bax; D2E11, cat. no. 5023, 1:1,000), anti-Bcl-2-associated death promoter (Bad; D24A9, cat. no. 9239, 1:1,000), anti-phosphorylated-Bad (Ser112) (40A9, cat. no. 5284, 1:1,000), anti-cytochrome $c$ (D18C7, cat. no. 11940, 1:1,000), anti-caspase-9 (cat. no. 9508, 1:1,000), anti-caspase-3 (cat. no. 9662; 1:1,000), anti-cleaved caspase-3 (cat. no. 9661; 1:1,000), anti-cyclooxygenase-IV (cat. no. 4844; $1: 1,000$ ), and anti- $\beta$-actin (cat. no. 4970; 1:2,000) overnight at $4^{\circ} \mathrm{C}$. The membranes were then washed three times with TBST for $10 \mathrm{~min}$, prior to being incubated for $1 \mathrm{~h}$ at room temperature with horseradish peroxidase-conjugated goat anti-rabbit (cat. no. 7074; 1:3,000) and horse anti-mouse (cat. no. 7076; 1:3,000) secondary antibodies. Following extensive washing, the proteins were detected using an enhanced chemiluminescence reagent (Merck Millipore). The band intensities were quantified using the ChemiDoc ${ }^{\mathrm{TM}} \mathrm{MP}$ system (Bio-Rad Laboratories, Inc., Hercules, CA, USA).

Reverse transcription-quantitative polymerase chain reaction $(R T-q P C R)$. Total RNA was extracted from the H9c2 cells using TRIzol reagent (Invitrogen Life Technologies, Carlsbad, CA, USA). RNA concentration was determined by UV spectrophotometry. cDNA was reverse transcribed from $1 \mu \mathrm{g}$ total RNA using ReverTra Ace- $\alpha^{\circledR}$ kit (Toyobo Co., Ltd., Osaka, Japan). A total of $2 \mu 1$ template cDNA was used for 
amplification, RT-qPCR was carried out using a Step One Plus $^{\text {TM }}$ Real-Time PCR system (Applied Biosystems Life Technologies, Foster City, CA, USA). The RT-qPCR was performed using Thunderbird SYBR Master Mix (Toyobo Co., Ltd., Osaka, Japan), and the following primer sequences were used: Bcl-2 forward, 5'-GGA GGA TTG TGG CCT TCT TTG-3', and reverse, 5'-CAT CCC AGC CTC CGT TAT CC-3' (annealing temperature $60^{\circ} \mathrm{C}$ ); Bax forward, 5'-GGT GAG CGA GGC GGT GAG GAC T-3', and reverse, 5'-GAG AGG ATG GCT GGG GAG AC-3' (annealing temperature $65^{\circ} \mathrm{C}$ ); Bad forward, 5'-TGA GGA AGA TGA AGG GAT GG-3', and reverse, 5'-GCT TTG TCG CAT CTG TGT TG-3' (annealing temperature $60^{\circ} \mathrm{C}$ ); caspase-9 forward, $5^{\prime}$-AGC TGG CCC AGT GTG AAT AC-3', and reverse, 5'-GCT CCC ACC TCA GTC AAC TC-3' (annealing temperature $60^{\circ} \mathrm{C}$ ); cytochrome $c$ forward, 5'-CTT GGG CTA GAG AGC GGG A-3', and reverse, 5'-GGT ATC CTC TCC CCA GGT GAT-3' (annealing temperature $60^{\circ} \mathrm{C}$ ); GAPDH forward, 5'-GAT CCC GCT AAC ATC AAA TG-3', and reverse, 5'-GAG GGA GTT GTC ATA TTT CTC-3' (annealing temperature $60^{\circ} \mathrm{C}$ ). All of the primers were designed and synthesized by GenScript Co., Ltd. (Nanjing, China). The RT-qPCR cycling conditions were as follows: $95^{\circ} \mathrm{C}$ for $10 \mathrm{~min}$, followed by $95^{\circ} \mathrm{C}$ for $15 \mathrm{sec}$, the respective primer annealing temperatures for $30 \mathrm{sec}$, and $72^{\circ} \mathrm{C}$ for $30 \mathrm{sec}$, and $95^{\circ} \mathrm{C}$ for $15 \mathrm{sec}$ for 40 cycles. GAPDH expression was used as an internal control. $2^{-\triangle \Delta C T}$ was calculated for every sample in order to determine the mRNA expression levels, which were normalized to GAPDH (27).

Measurement of mitochondrial membrane potential ( $\Delta \Psi m)$. The mitochondria of $\mathrm{H} 9 \mathrm{c} 2$ cells were stained with JC-1. A total of $1 \times 10^{6}$ cells were incubated with $10 \mu \mathrm{g} / \mathrm{ml} \mathrm{JC}-1$ (Nanjing Keygene Biotech Co., Ltd., Nanjing, China) dissolved in DMEM without FBS at $37^{\circ} \mathrm{C}$ for $30 \mathrm{~min}$, then washed three times with PBS. The fluorescence of the cells was measured at an excitation wavelength of $507 \mathrm{~nm}$ and an emission wavelength of $529 \mathrm{~nm}$ using a Safire2 microplate reader (Tecan Schweiz AG).

Statistical analysis. Statistical analyses were performed using SPSS 10.0 (SPSS, Inc., Chicago, IL, USA). Data are expressed as the mean \pm standard deviation. A one-way analysis of variance was performed when $>2$ groups were compared, and when statistically significant a Student-Newman-Keuls test was used to investigate the differences between individual groups. $\mathrm{P}<0.05$ was considered to indicate a statistically significant difference.

\section{Results}

BPV exhibits inhibitory effects on H9c2 myoblast cell proliferation. The results of the cell viability assay are shown in Fig. 1. The H9c2 myoblast cells were treated with $1 \mathrm{mM}$ BPV over a time course of 12, 24 and $36 \mathrm{~h}$. The cell viability levels at the $12 \mathrm{~h}$ time point decreased to $81.32 \pm 2.92 \%$, as compared with the control cells. At $24 \mathrm{~h}$, an increase in BPV-induced inhibition of cell proliferation was observed $(51.23 \pm 2.15 \%)$, whereas at $36 \mathrm{~h}$, cell viability was further reduced to $24.57 \pm 1.58 \%$. These results suggest that BPV exerted inhibitory effects on $\mathrm{H} 9 \mathrm{c} 2$ myoblast cell growth in a time-dependent manner.
LE treatment exhibits rescue effects on $B P V$-induced $H 9 c 2$ myoblast cell apoptosis. BPV-induced apoptosis of the $\mathrm{H} 9 \mathrm{c} 2$ myoblast cells was investigated using Annexin V/PI staining assay. As shown in Fig. 2A and B, the apoptotic rate of the $\mathrm{H} 9 \mathrm{c} 2$ myoblast cells was measured for $24 \mathrm{~h}$ following cellular treatment with BPV or LE alone, and following co-treatment with BPV and LE. The percentage of apoptotic cells was $4.54 \pm 0.20 \%$ for the untreated control group, whereas the cells treated with $1 \mathrm{mM}$ BPV exhibited $24.66 \pm 2.57 \%$ apoptosis, including both early- and late-stage apoptotic cells. When the H9c2 myoblast cells were treated with $1 \mathrm{mM}$ BPV combined with $1 \%$ LE, LE was able to attenuate the apoptotic effects induced by BPV treatment, and the co-treated cell apoptotic levels resembled those of the control group. The addition of LE to the BPV-treated group reduced the apoptotic rate to a

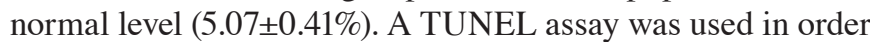
to investigate the apoptotic cells. As shown in Fig. $2 \mathrm{C}$, the H9c2 myoblast cells exhibited extensive apoptosis following BPV treatment. However, LE treatment lowered BPV-induced apoptosis. These results suggest that co-treatment with BPV and LE may protect $\mathrm{H} 9 \mathrm{c} 2$ myoblast cells from apoptosis.

$L E$ treatment reverses the effects of $B P V$ on the Bcl-2 and caspase families. Bcl-2 and caspase are two protein families that have a crucial role in cell apoptosis. In the present study, western blotting and RT-qPCR were used to investigate the changes in protein and mRNA expression levels of Bcl-2 and caspase. The results of the western blot analysis revealed that the protein expression levels of Bax, cleaved caspase 9, and cleaved caspase 3 were elevated in the BPV-treated cells, whereas the protein expression levels of Bcl-2 and phosphorylated-Bad were downregulated. However, co-treatment of the H9c2 myoblast cells with LE and BPV resulted in opposing results (Fig. 3A and B). The downregulated expression levels of Bcl-2 and phosphorylated-Bad were restored to normal, and similarly the expression levels of cleaved caspase- 3 and -9 were reduced to that of the control sample. RT-qPCR was performed in order to verify whether Bcl-2 and Bax were transcriptionally regulated. The results of the RT-qPCR confirmed that the mRNA expression levels of Bcl-2 and Bax were also regulated by BPV and $\mathrm{LE}$ in a similar manner to the protein expression levels (Fig 3C). In addition, the ratio of Bax/Bcl-2 doubled following BPV treatment, indicating that the apoptotic levels has increased. This ratio returned to normal in the BPV and LE co-treated H9c2 myoblast cells (Fig. 3C and D). These results indicate that BPV and LE may regulate apoptosis via $\mathrm{Bcl}-2$ and caspase at both the protein and mRNA level.

LE treatment attenuates BPV-triggered oxidative stress in H9c2 myoblast cells. Oxidative stress is caused by disturbance to the oxidation and antioxidation balance, leading to apoptotic cell death (28). In order to investigate whether oxidative stress mediated the effects of BPV and LE, the activity levels of various enzymes were monitored. ROS levels, the accumulation of which may lead to mitochondrial dysfunction, were detected using a DCFH-DA probe. The results indicated that a rapid production of ROS occurred following $\mathrm{H} 9 \mathrm{c} 2$ myoblast cell exposure to BPV. However, BPV-induced ROS production was reversed by LE treatment $(127.54 \pm 3.68 \%$ in the BPV group, vs. 104.33 $\pm 2.77 \%$ in the BPV+LE group, 


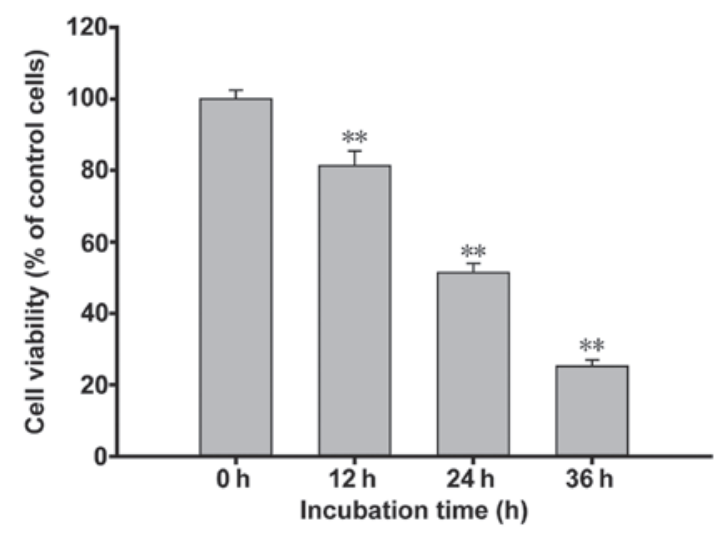

Figure 1. Inhibitory effects of bupivacaine (BPV) on H9c2 myoblast cell viability. The H9c2 rat myoblast cells were treated with $1 \mathrm{mM}$ BPV for 12,24 , and $36 \mathrm{~h}$. Cell viability was determined using an MTT assay. Data are presented as the mean \pm standard deviation. All experiments were performed in triplicate. ${ }^{* *} \mathrm{P}<0.01$, vs. the control group.

A

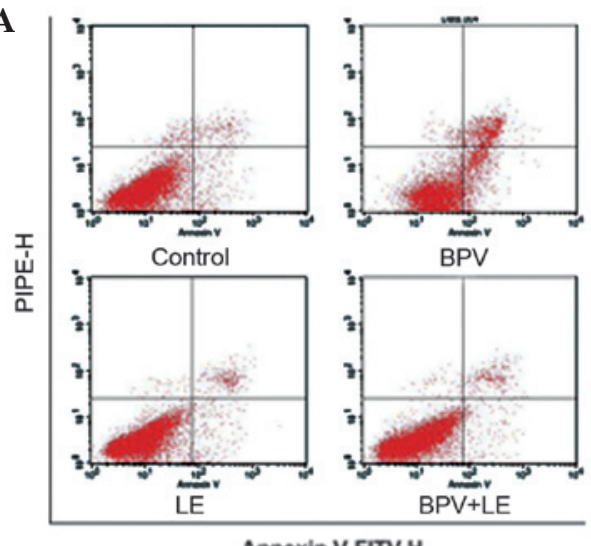

C
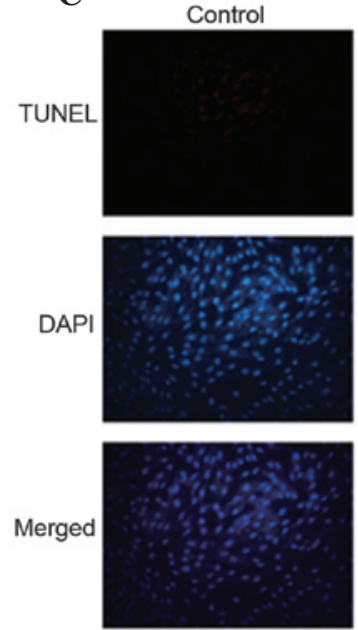

BPV
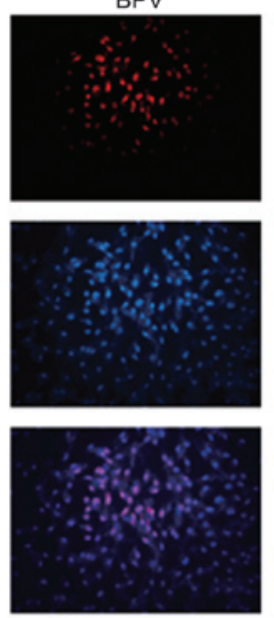

B

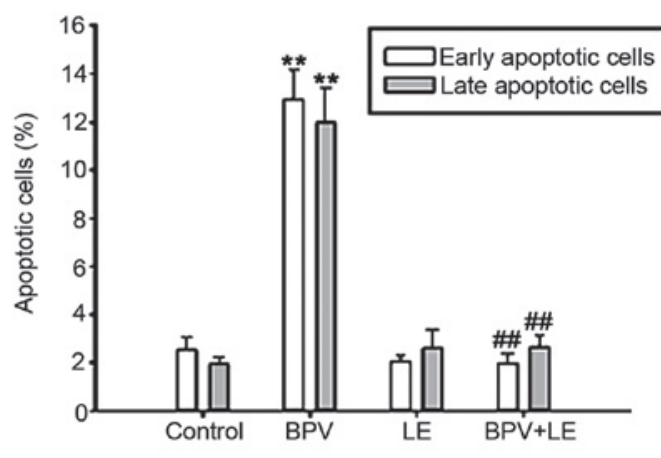

LE
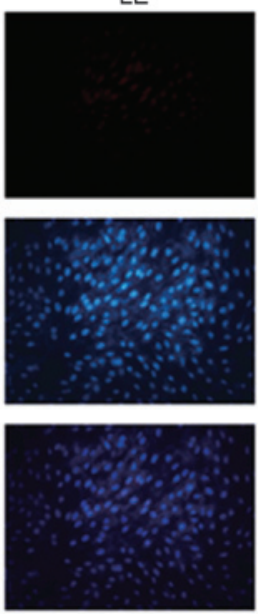

BPV+LE
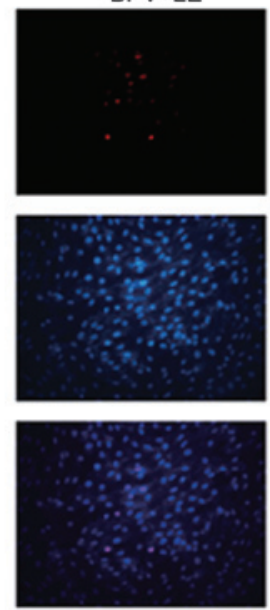

Figure 2. Lipid emulsion (LE) treatment reversed bupivacaine (BPV)-induced apoptosis of H9c2 myoblast cells. The H9c2 rat myoblast cells were treated with $1 \mathrm{mM}$ BPV for $24 \mathrm{~h}$. (A) Annexin V/propidium iodide double staining assay of the H9c2 myoblast cells was analyzed by flow cytometry. (B) The BPVinduced_apoptotic rate of the $\mathrm{H} 9 \mathrm{c} 2$ myoblast cells was plotted for both the early and late stages of apoptosis. (C) Immunofluorescent detection of DNA damage (TUNEL) in H9c2 myoblast cells. Following treatment, the H9c2 myoblast cells were fixed and processed for TUNEL (red) assay. The nuclei were stained with 4',6-diamidino-2-phenylindole (DAPI; blue) (magnification, x200). All data were presented as the mean \pm standard deviation, and all experiments were performed in triplicate. ${ }^{* *} \mathrm{P}<0.01$, vs. the control group; ${ }^{\# \prime} \mathrm{P}<0.01$, vs. the $1 \mathrm{mmol} / 1 \mathrm{BPV}$ treatment group.

Fig. 4A). MDA is a marker for lipid peroxidation, which was used as a further indicator of endogenous ROS levels. The BPV-treated cell samples contained elevated levels of MDA
$(61.33 \pm 3.43 \mu \mathrm{mol} / \mathrm{mg})$, as compared with the control group $(41.86 \pm 0.64 \mu \mathrm{mol} / \mathrm{mg}$ ) (Fig. 4B). These results suggest that BPV treatment causes apoptotic cell death by increasing 

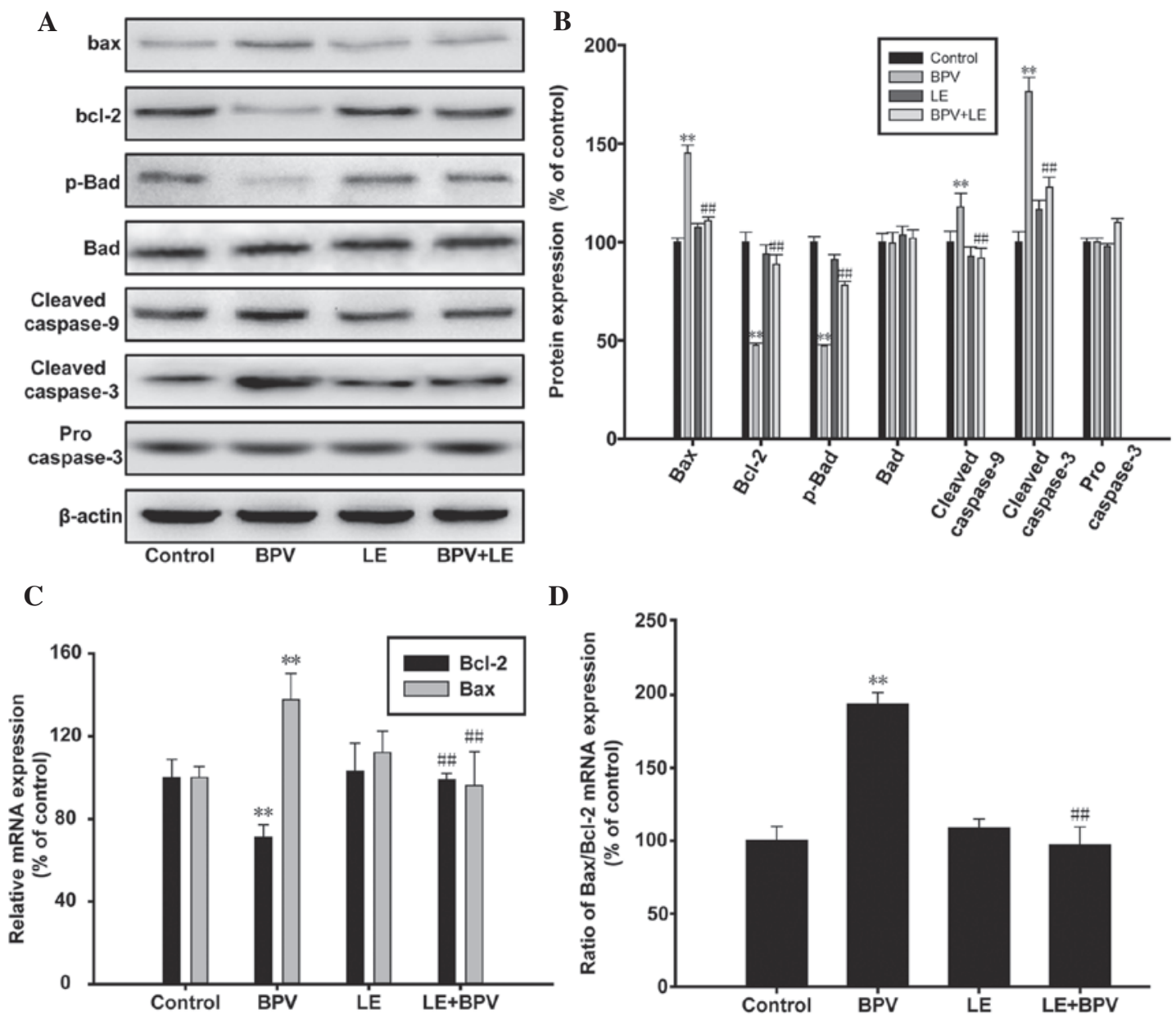

Figure 3. Effects of bupivacaine (BPV) on B-cell lymphoma 2 (Bcl-2) and caspase. (A and B) The H9c2 rat myoblast cells were treated with $1 \mathrm{mM}$ BPV or $1 \%$ lipid emulsion (LE) alone, or co-treated with both BPV and LE for $24 \mathrm{~h}$, prior to the collection of the cell lysate. Western blot analysis was performed using antibodies targeting Bcl-2, Bcl-2-associated X protein (Bax), phosphorylated (p)-Bcl-2-associated death promoter (Bad), Bad, cleaved caspase 3, cleaved caspase 9 and procasepase 3. (C and D) The mRNA expression levels of Bcl-2 and Bax in the H9c2 myoblast cells were quantified using reverse transcription-quantitative polymerase chain reaction. The data are presented as the mean \pm standard deviation, and all experiments were performed in tripliacate. ${ }^{* *} \mathrm{P}<0.01$, vs. the control group; ${ }^{\# \#} \mathrm{P}<0.01$, vs. the $1 \mathrm{mM}$ BPV treatment group.

cellular ROS levels. In order to further verify this hypothesis, the activity levels of SOD were investigated. SOD antioxidant activity was measured by WST-1. The activity levels of SOD were significantly reduced following BPV treatment (Fig. 4C). In addition, co-treatment of the H9c2 myoblast cells with LE restored SOD activity levels to those of the control (74.03 $\pm 6.23 \%$ in the BPV-treated sample, vs. $92.83 \pm 5.81 \%$ in the BPV+LE co-treated sample; Fig. 4C). CAT activity, which is another key indicator of endogenous ROS levels, was measured in order to assess the levels of antioxidation within the H9c2 myoblast cells. As shown in Fig. 4D, the CAT activity levels exhibited a similar trend to SOD activity levels. CAT activity was reduced in the BPV-treated cells, whereas co-treatment with LE restored CAT activity levels to those of the control group $(709.74 \pm 22.40 \mathrm{U} / \mathrm{mg}$ in the control group, $467.03 \pm 25.39 \mathrm{U} / \mathrm{mg}$ in the $\mathrm{BPV}$-treated group, and $650.47 \pm 19.430 \mathrm{U} / \mathrm{mg}$ in the BPV+LE co-treated group). These results suggest that BPV-induced toxicity is attributable to an increase in the oxidative stress levels of $\mathrm{H} 9 \mathrm{c} 2$ myoblast cells. Furthermore, co-treatment with BPV and LE reversed the observed increase in cellular oxidation, bringing the levels of endogenous ROS back to normal.
$L E$ reverses $B P V$-induced apoptosis by promoting mitochondrial survival. Two major pathways trigger apoptotic cell death: The death receptor-induced extrinsic pathway, and the mitochondrial apoptosome-mediated apoptotic intrinsic pathway (29). The mitochondrial membrane potential $(\Delta \Psi)$-sensitive dye JC-1 was used in order to determine through which pathway BPV and LE affect $\mathrm{H} 9 \mathrm{c} 2$ myoblast cell survival. When mitochondrial $\Delta \Psi$ is high, JC-1 accumulates in the mitochondrial matrix, and forms red fluorescent JC-1 aggregates. However, when mitochondrial $\Delta \Psi$ is low, JC-1 becomes a monomer and emits green fluorescence. The ratio of green to red fluorescence indicates the percentage of mitochondrial depolarization. Flow cytometric analysis revealed that the $\mathrm{H} 9 \mathrm{c} 2$ myoblast cells became more susceptible to mitochondrial membrane depolarization following BPV treatment, as compared with those in the control group (Fig. 5A). The percentage of green fluorescence increased from $3.21 \pm 0.91 \%$ to $36.34 \pm 3.40 \%$ following BPV treatment, indicating a decrease in mitochondrial $\Delta \Psi$, and mitochondrial dysfunction. The percentage of JC-1 monomers also decreased significantly in the cells co-treated with BPV and LE $(12.22 \pm 1.63 \%)$. mPTP opening promotes uncontrolled 
A

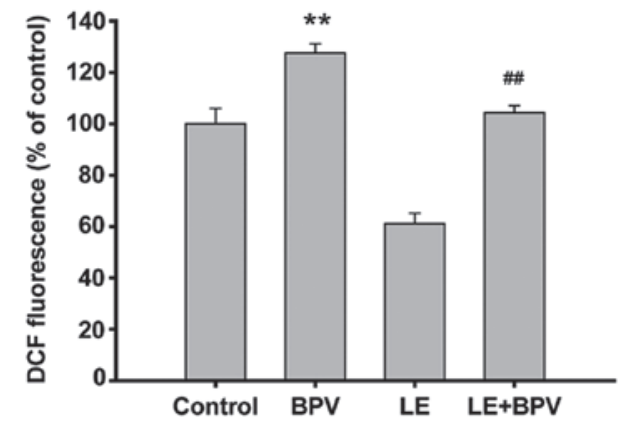

C

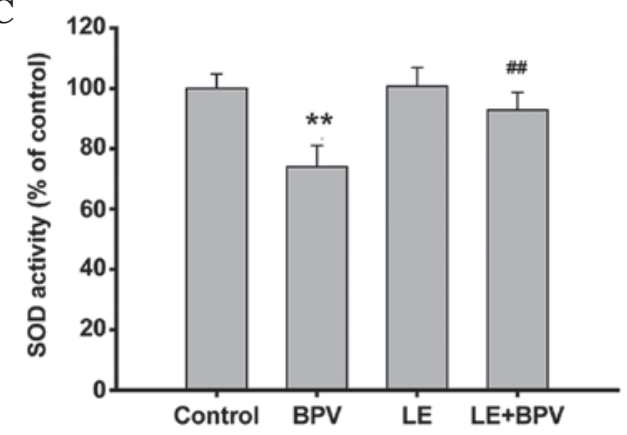

B

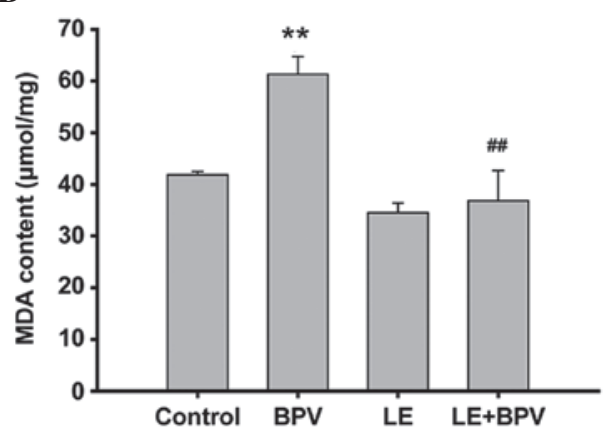

D

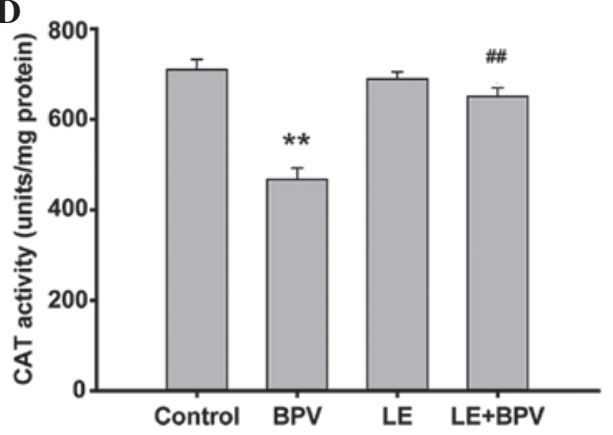

Figure 4. Effects of lipid emulsion (LE) and bupivacaine (BPV) treatment on the oxidative stress of H9c2 myoblast cells. (A) Endogenous levels of reactive oxygen species in the $\mathrm{H} 9 \mathrm{c} 2$ rat myoblast cells were measured by dichloro-dihydro-flurorescein (DCF) diacetate fluorescence in the control, the 1 mM BPV-treated, the 1\% LE-treated, and the BPV and LE co-treated groups. (B) The concentration of malondialdehyde (MAD) in the H9c2 myoblast cells was measured and compared among the four different treatment groups. (C) The superoxide dismutase (SOD) activity levels were measured in the H9c2 myoblast cells and compared to the control untreated cells. (D) The catalase (CAT) activity levels in the H9c2 myoblast cells were quantified and compared among the four different treatment groups. The data are presented as the mean \pm standard deviation, and all experiments were performed in triplicate. ${ }^{* *} \mathrm{P}<0.01$, vs. the

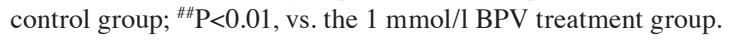

ion flow between the mitochondrial membranes, which leads to mitochondrial swelling and rupture, followed by the release of cytochrome $c$ (30). Therefore, the levels of cytochrome $c$ reflect mPTP state, which provides an indication of mitochondrial viability. In the present study, cytochrome $c$ release within the mitochondria was quantified via immunoblotting. The results demonstrated that treatment with BPV significantly reduced mitochondrial expression levels of cytochrome $c$, whereas BPV treatment significantly increased cytosolic cytochrome $c$ expression levels (Fig. 5B and $\mathrm{C}$ ). The addition of LE to the BPV-treated cells reduced the cytosolic expression levels of cytochrome $c$ similar to those observed in the control cells. CsA treatment also inhibited cytochrome $c$ release in the BPV-treated cells. Cellular treatment with Atr eliminated the reversal effects induced by LE treatment. These results strongly support the hypothesis that LE counteracts the cellular toxicity induced by treatment with BPV. Furthermore, the results of the present study suggest that the molecular mechanism underlying the protective effects of LE on $\mathrm{H} 9 \mathrm{c} 2$ myoblast cells is modulated via the mPTP.

\section{Discussion}

Previous studies have demonstrated that BPV exhibits cellular toxicity both in vitro and in vivo (15-17). However, LE treatment is able to reverse BPV-induced cardiotoxicity. Chen et al (31) recently reported that BPV-induced asystole may be reversed by LE in rat hearts. Rosenblatt et al (32) also reported the successful clinical use of LE to resuscitate patients following BPV-associated cardiac arrest. However, the mechanism underlying BPV toxicity and the protective effects of LE have yet to be elucidated.

In the present study, the MTT assay demonstrated that BPV inhibited cellular proliferation in a time-dependent manner. The results of Annexin V/PI staining indicated that BPV induced cellular apoptosis, and the results of the TUNEL assay further confirmed this observation. The present study successfully demonstrated that LE exhibited protective effects on $\mathrm{H} 9 \mathrm{c} 2$ myoblast cells, and rescued them from BPV-induced apoptosis.

Oxidative stress is closely associated with apoptosis. Previous studies have demonstrated that ROS and oxidative stress have an important role in the onset of apoptosis. Antioxidants and thiol reductants, such as $\mathrm{N}$-acetylcysteine, as well as the overexpression of manganese-SOD are able to prevent or delay apoptosis (33). The present study investigated changes in the activity levels of ROS and antioxidants, and demonstrated that treatment with BPV caused oxidative stress. Incubation of the H9c2 myoblast cells with BPV for $24 \mathrm{~h}$ resulted in elevated levels of ROS and MDA, and reduced levels of SOD and CAT. Treatment with LE counteracted BPV-induced cell death caused by ROS-induced cellular damage. The results of the present study suggested that BPV-induced cellular apoptosis may be closely associated with oxidative stress. Furthermore, the rescue effects observed following LE treatment are likely attributable to an increase in the levels of antioxidants. 
A

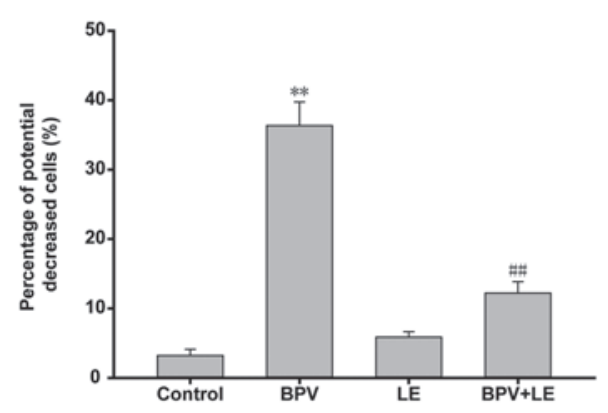

B

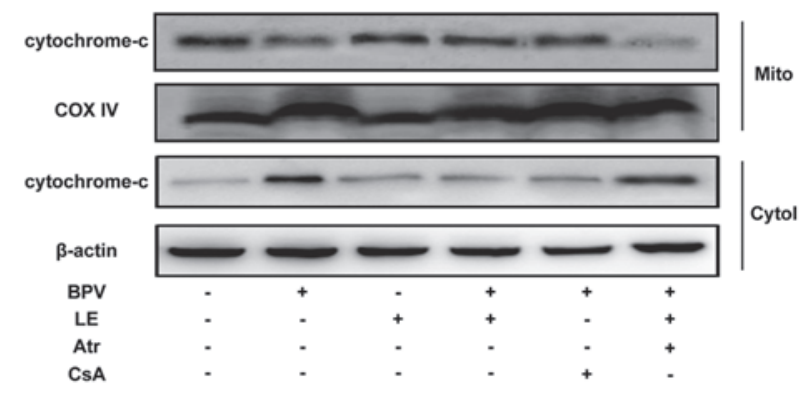

C

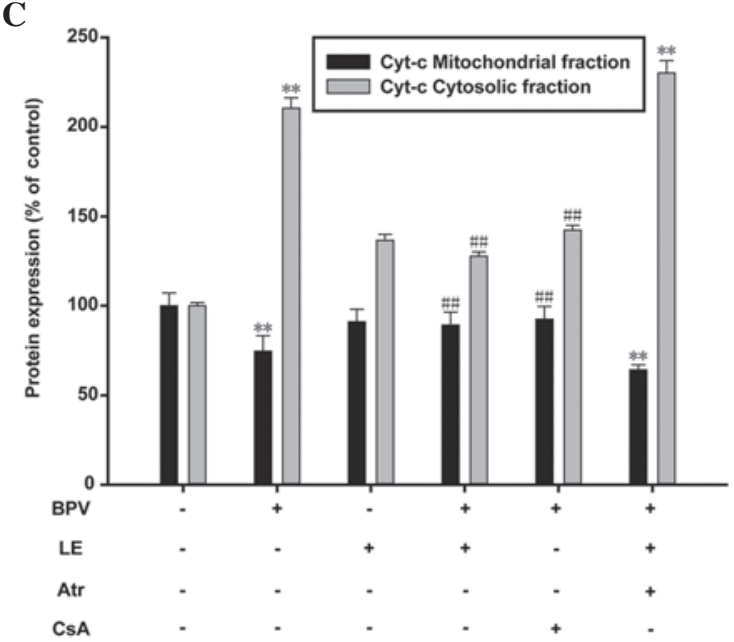

Figure 5. Lipid emulsion (LE) treatment rescued bupivacaine (BPV)-induced apoptosis by mediating mitochondrial viability. (A) The H9c2 rat myoblast cells were exposed to $1 \mathrm{mM} \mathrm{BPV}$ or $1 \% \mathrm{LE}$, or co-treated with both BPV and LE for $24 \mathrm{~h}$, prior to being stained with JC-1. The percentage of H9c2 myoblast cells with a collapsed membrane potential was analyzed by flow cytometry. (B and C) The H9c2 myoblast cells were treated with $1 \mathrm{mM} \mathrm{BPV}, 1 \%$ LE, cyclosporin A (CsA), or atractyloside (Atr) at various concentrations for $24 \mathrm{~h}$. The mitochondrial and cytosolic fractions were then subjected to western blot analysis. The data are presented as the mean \pm standard deviation, and all experiments were performed in triplicated. ${ }^{* *} \mathrm{P}<0.01$, vs. the control; ${ }^{\# \#} \mathrm{P}<0.01$, vs. the $1 \mathrm{mM} B P V$ treatment group. COX, cyclooxygenase.

The present study investigated the effects of various apoptosis-associated proteins, and revealed that BPV and LE influence the expression levels of Bcl-2 and caspase family proteins. The results of the present study showed that treatment with BPV increased the protein expression levels of Bax, and inhibited the protein expression levels of Bcl-2. RT-qPCR further demonstrated that the mRNA expression levels of Bax and Bcl-2 were affected. In addition, treatment with BPV activated caspases-9 and -3. The upregulation of apoptotic proteins following BPV treatment was rescued by LE treatment. The ratio of $\mathrm{Bax} / \mathrm{Bcl}-2$ is representative of the severity of mitochondrial outer membrane permeabilization, which is a critical factor of the intrinsic apoptotic pathway (34-36). These results support the hypothesis that the mitochondria are critical in cellular apoptosis. Furthermore, mitochondria have been implicated in the generation of ROS (37). These previous results are concordant with the results of the present study, and suggest that BPV-induced toxicity directly affects mitochondrial survival.

In order to further confirm this hypothesis, and to elucidate the molecular mechanism underlying the rescue effects induced by LE treatment, a series of experiments were carried out. The results of JC-1 staining indicated that treatment with BPV affected H9c2 myoblast cell survival by initiating mitochondrial dysfunction. Release of cytochrome $c$ from the mitochondria into the cytosol also occurred following BPV treatment, and was determined to be a key marker of apoptosis. Mitochondrial function and the release of cytochrome $c$ were inversely correlated to LE treatment. MPTP, which is a non-specific pore located in the inner mitochondrial membrane, is responsible for the release of cytochrome $c$. The effects of BPV and LE on MPTP are associated with the levels of cytochrome $c$ release. In addition, the effects of CsA and Atr, an mPTP inhibitor and activator respectively, on the release of cytochrome $c$ were compared with that of BPV and LE. The results demonstrated that MPTP has a significant role in the mechanism underlying the protective effects of LE on BPV-induced toxicity. The results of the present study confirmed the involvement of the mitochondria in BPV-induced toxicity, and the important role of mPTP in the mechanism underlying the protective effects of LE treatment.

The present study demonstrated the potential clinical applications of LE as a treatment for BPV-induced toxicity. Furthermore, the present study revealed that the mechanism underlying BPV-induced toxicity is associated with the mitochondria, via the alteration of mPTP sensitivity. In conclusion, LE may protect $\mathrm{H} 9 \mathrm{c} 2$ myoblast cells from 
BPV-induced toxicity through restoration of mitochondrial function.

\section{Acknowledgements}

The present study was supported by grants from the National Natural Science Foundation of China (grant no. 81360284), and from the Natural Science Foundation of Ningxia (grant no. NZ13148).

\section{References}

1. Malhotra N, Chanana C, Roy KK, Kumar S, Rewari V and Sharma JB: To compare the efficacy of two doses of intraperitoneal bupivacaine for pain relief after operative laparoscopy in gynecology. Arch Gynecol Obstet 276: 323-326, 2007.

2. Clarkson CW and Hondeghem LM: Mechanism for bupivacaine depression of cardiac conduction: Fast block of sodium channels during the action potential with slow recovery from block during diastole. Anesthesiology 62: 396-405, 1985.

3. Lynch C III: Depression of myocardial contractility in vitro by bupivacaine, etidocaine, and lidocaine. Anesth Analg 65: 551-559, 1986

4. Castle NA: Bupivacaine inhibits the transient outward $\mathrm{K}^{+}$ current but not the inward rectifier in rat ventricular myocytes. J Pharmacol Exp Ther 255: 1038-1046, 1990.

5. Halestrap AP, McStay GP and Clarke SJ: The permeability transition pore complex: Another view. Biochimie 84: 153-166, 2002

6. Halestrap AP, Clarke SJ and Khaliulin I: The role of mitochondria in protection of the heart by preconditioning. Biochim Biophys Acta 1767: 1007-1031, 2007.

7. Bernardi P, Krauskopf A, Basso E, Petronilli V, Blachly-Dyson E, Di Lisa F and Forte MA: The mitochondrial permeability transition from in vitro artifact to disease target. FEBS J 273: 2077-2099, 2006.

8. Halestrap AP, Clarke SJ and Javadov SA: Mitochondrial permeability transition pore opening during myocardial reperfusion - a target for cardioprotection. Cardiovasc Res 61: 372-385, 2004.

9. Stone JR and Yang S: Hydrogen peroxide: A signaling messenger. Antioxid Redox Signal 8: 243-270, 2006.

10. Del Rio D, Stewart AJ and Pellegrini N: A review of recent studies on malondialdehyde as toxic molecule and biological marker of oxidative stress. Nutr Metab Cardiovasc Dis 15: 316-328, 2005.

11. Wu JQ, Kosten TR and Zhang XY: Free radicals, antioxidant defense systems, and schizophrenia.Prog Neuropsychopharmacol Biol Psychiatry 46: 200-206, 2013.

12. Gałecka E, Jacewicz R, Mrowicka M, Florkowski A and Gałecki P: Antioxidative enzymes - structure, properties, functions. Pol Merkur Lekarski 25: 266-268, 2008 (In Polish).

13. Bowler C, Slooten L, Vandenbranden S, De Rycke R, Botterman J, Sybesma C, Van Montagu M and Inzé D: Manganese superoxide dismutase can reduce cellular damage mediated by oxygen radicals in transgenic plants. EMBO J 10: 1723-1732, 1991.

14. Long WB, Rosenblum S and Grady IP: Successful resuscitation of bupivacaine-induced cardiac arrest using cardiopulmonary bypass. Anesth Analg 69: 403-406, 1989.

15. Weinberg G, Ripper R, Feinstein DL and Hoffman W: Lipid emulsion infusion rescues dogs from bupivacaine-induced cardiac toxicity. Reg Anesth Pain Med 28: 198-202, 2003.

16. Weinberg GL, VadeBoncouer T, Ramaraju GA, Garcia-AmaroMF and Cwik MJ: Pretreatment or resuscitation with a lipid infusion shifts the dose-response to bupivacaine-induced asystole in rats. Anesthesiology 88: 1071-1075, 1998.

17. Weinberg GL, Ripper R, Murphy P, Edelman LP, Hoffman W, Strichartz G and Feinstein DH: Lipid infusion accelerates removal of bupivacaine and recovery from bupivacaine toxicity in the isolated rat heart. Reg Anesth Pain Med 31: 296-303, 2006
18. Weinberg GL: Lipid infusion therapy: Translation to clinical practice. Anesth Analg 106: 1340-1342, 2008.

19. Litz RJ, Roessel T, Heller AR and Stehr SN: Reversal of central nervous system and cardiac toxicity after local anesthetic intoxication by lipid emulsion injection. Anesth Analg 106: 1575-1577, 2008.

20. Weinberg GL, Palmer JW, VadeBoncouer TR, Zuechner MB, Edelman G and Hoppel CL: Bupivacaine inhibits acylcarnitine exchange in cardiac mitochondria. Anesthesiology 92: 523-528, 2000.

21. Daoud A, Song J, Xiao F and Shang J: B-9-3, a novel $\beta$-carboline derivative exhibits anti-cancer activity via induction of apoptosis and inhibition of cell migration in vitro. Eur J Pharmacol 724: 219-230, 2014.

22. Tian YY, An LJ, Jiang L, Duan YL, Chen J and Jiang B: Catalpol protects dopaminergic neurons from LPS-induced neurotoxicity in mesencephalic neuron-glia cultures. Life Sci 80: 193-199, 2006.

23. Martin RC, Liu Q, Wo JM, Ray MB and Li Y: Chemoprevention of carcinogenic progression to esophageal adenocarcinoma by the manganese superoxide dismutase supplementation. Clin Cancer Res 13: 5176-5182, 2007.

24. Qian J, Jiang F, Wang B, Yu Y, Zhang X, Yin Z and Liu C: Ophiopogonin D prevents $\mathrm{H}_{2} \mathrm{O}_{2}$-induced injury in primary human umbilical vein endothelial cells. J Ethnopharmacol 128: 438-445, 2010.

25. He Z, Yu S, Mei G, Zheng M, Wang M, Dai Y, Tang B and Li N: Maternally transmitted milk containing recombinant human catalase provides protection against oxidation for mouse offspring during lactation. Free Radic Biol Med 45: 1135-1142, 2008.

26. Zhang Y, Yan M, Yu A, Mao H and Zhang J: Inhibitory effects of $\beta$-tricalciumphosphate wear particles on osteocytes via apoptotic response and Akt inactivation. Toxicology 297: 57-67, 2012.

27. Livak KJ and Schmittgen TD: Analysis of relative gene expression data using real-time quantitative PCR and the 2(-Delta Delta C(T)) Method. Methods 25: 402-408, 2001.

28. Sies H and Cadenas E: Oxidative stress: Damage to intact cells and organs. Philos Trans R Soc Lond B Biol Sci 311: 617-631, 1985.

29. Hu W and Kavanagh JJ: Anticancer therapy targeting the apoptotic pathway. Lancet Oncol 4: 721-729, 2003.

30. Halestrap A: Biochemistry: A pore way to die. Nature 434: 578-579, 2005.

31. Chen Y, Xia Y, Liu L, Shi T, Wang Q, Chen L, Papdimos TJ and $\mathrm{Xu} \mathrm{X}$ : Lipid emulsion reverses bupivacaine-induced asystole in isolated rat hearts: Concentration-response and time-response relationships. Anesthesiology 113: 1320-1325, 2010.

32. Rosenblatt MA, Abel M, Fischer GW, Itzkovich CJ and Eisenkraft JB: Successful use of a $20 \%$ lipid emulsion to resuscitate a patient after a presumed bupivacaine-related cardiac arrest. Anesthesiology 105: 217-218, 2006.

33. Kannan K and Jain SK: Oxidative stress and apoptosis. Pathophysiology 7: 153-163, 2000.

34. Osorio LM, De Santiago A, Aguilar-Santelises M, Mellstedt H and Jondal M: CD6 ligation modulates the Bcl-2/Bax ratio and protects chronic lymphocytic leukemia B cells from apoptosis induced by anti-IgM. Blood 89: 2833-2841, 1997.

35. Del Poeta G, Venditti A, Del Principe MI, Maurillo L, Buccisano F, Tamburini A, Cox MC, Franchi A, Bruno A, Mazzone C, et al: Amount of spontaneous apoptosis detected by $\mathrm{Bax} / \mathrm{Bcl}-2$ ratio predicts outcome in acute myeloid leukemia (AML). Blood 101: 2125-2131, 2003.

36. Cory S, Huang DC and Adams JM: The Bcl-2 family: Roles in cell survival and oncogenesis. Oncogene 22: 8590-8607, 2003.

37. Kulbacka J, Bar J, Chwilkowska A, Dumanska M, Drag-Zalesinska M, Wysoka T, Stach H, Becharz I, Lugowski M, Markinkowska A, et al: Oxidative modulation of marcaine and lekoptin in H9C2 rat myoblasts. Acta Pharmacol Sin 30: 184-192, 2009. 